\title{
Clinical Characteristics of Pediatric Patients with Congenital Erythrocytosis: A Single Center Study
}

\author{
Sema Aylan, Gelen ${ }^{1}$, Nazan Sarper ${ }^{1}$, Emine Zengin ${ }^{2}$, İnci Tahsin ${ }^{3}$, and Mehmet Azizoğlu ${ }^{2}$ \\ ${ }^{1}$ Kocaeli Univ. Faculty of Medicine \\ ${ }^{2}$ Kocaeli University \\ ${ }^{3}$ Kocaeli Üniversitesi Tıp Fakültesi
}

April 12, 2021

\begin{abstract}
Background: Although congenital erythrocytosis $(\mathrm{CE})$, an inherited disorder, impairs pediatric quality of life, physicians often overlook high hemoglobin ( $\mathrm{Hgb}$ ) levels and its symptoms due to lack of knowledge of age-adjusted pediatric Hgb levels and CE's rarity. Methods: In a retrospective, single-center study, data from hospital records of patients ( $<18$ years) diagnosed with CE were evaluated. Results: Forty patients from 39 families (male: female ratio $=7: 1$ ) had been diagnosed with CE in a 20-year period, at a mean age of $15.31 \pm 2.49$ years (8.34-17.92) and with mean Hgb levels of $17.4 \pm 1.34 \mathrm{~g} / \mathrm{dL}(14.63-23.0)$. No serum erythropoietin levels exceeded the reference levels. Although the most common symptom was headache (80.0\%), $40.0 \%$ of patients presented with at least one gastrointestinal symptom (e.g., nausea, vomiting, abdominal pain, and rectal bleeding), and $75.0 \%$ exhibited plethora. None had leukocytosis, thrombocytosis, abnormal capillary oxygen saturation, JAK2 mutation, and venous blood gas analysis and Hgb electrophoresis revealed no abnormalities. While $43.6 \%$ of patients had family histories of CE, $28.2 \%$ had 15-48-year-old relatives who had experienced myocardial infarction, stroke, and/or sudden death. Six asymptomatic patients were detected incidentally. When symptoms of hyperviscosity were present, aspirin was prescribed, and phlebotomy performed. No thrombotic episodes occurred as a result. Conclusion: To detect CE, physicians should assess Hgb levels in consideration of normal age-adjusted levels in children. Pediatric patients with CE may also present with gastrointestinal symptoms. Although no thrombotic episode occurred among the patients, their family histories included life-threatening thrombotic episodes, even in adolescents.
\end{abstract}

\section{INTRODUCTION}

Erythrocytosis is defined as hemoglobin (Hgb) and hematocrit (Hct) levels exceeding $16 \mathrm{~g} / \mathrm{dL}$ and $48 \%$ in women and $16.5 \mathrm{~g} / \mathrm{dL}$ and $49 \%$ in men, both respectively. In children, a Hgb level exceeding the normal reference range in the age- and gender-adjusted 99th percentile, or an increase of $2 \mathrm{~g} / \mathrm{dL}$ in Hgb level from the baseline in at least two whole blood counts at different times, is considered to indicate erythrocytosis. ${ }^{1-3}$ A red cell mass greater than $125 \%$ of the mass predicted for sex and body mass also indicates erythrocytosis. ${ }^{4}$

In childhood, erythrocytosis is either familial or secondary to cardiopulmonary disease that causes a hypoxiainduced rise in erythropoietin (EPO). Among familial cases, congenital erythrocytosis (CE) is an autosomaldominant or recessively inherited disorder that enhances EPO's effect, as seen in erythropoietin receptor (EPOR) mutations. In EPOR mutations, erythrocytosis is due to primary cause that is intrinsic to red cells. EPOR plays a role in stimulating the proliferation of erythroid progenitor cells and preventing their apoptosis. ${ }^{5-8}$

Other cases of familial erythrocytosis are secondary and due to dysregulated EPO production resulting from defects in oxygen-sensing pathways. Some mutations in the globin chains of Hgb raise the affinity of Hgb 
molecules for oxygen, which impairs the normal loading of oxygen in the lungs and the delivery of oxygen to tissues. In Hgb variants with high oxygen affinity, serum EPO is elevated, while $\mathrm{pO} 2$ and oxygen saturation is normal due to compensatory erythrocytosis. Certain mutations may also decrease the affinity of $\mathrm{Hgb}$ for $2,3-$ bisphosphoglycerate mutase and cause erythrocytosis. Although Hgb electrophoresis with high-performance liquid chromatography, the currently preferred method, cannot detect all variants, sequencing the globin DNA can enable the detection of such mutations. Oxygen pressure at $50 \%$ Hgb saturation $\left(\mathrm{P}_{50}\right)$ is low in the presence of high oxygen affinity Hgb variants, congenital methemoglobinemia, 2,3-bisphosphoglycerate mutase deficiency, and other rare conditions. ${ }^{7,8}$

In patients with erythrocytosis who also experience leukocytosis, thrombocytosis, and splenomegaly, polycythemia vera (PV) may be the correct diagnosis. PV is a myeloproliferative disease rarely seen in childhood in which erythrocytosis is autonomous and EPO production is low or nonexistent. ${ }^{78}$ In erythrocytosis, elevated serum EPO levels or findings of chronic hypoxia (e.g., cyanosis, dyspnea, and finger clubbing) suggest secondary causes. ${ }^{5}$ Along with cardiac and pulmonary disease, living at a high altitude, smoking tobacco, carbon monoxide poisoning, methemoglobinemia, and obstructive sleep apnea syndrome rank among secondary causes of erythrocytosis. Beyond that, androgen preparations, renal artery stenosis, post-renal transplantation, renal cysts, renal cell carcinoma, cerebellar PV, meningioma, parathyroid adenoma, pheochromocytoma, and hepatocellular carcinoma may cause inappropriate EPO production and, in turn, erythrocytosis. ${ }^{5,6,9}$

In EPOR mutations, abnormality is limited to erythroid series in whole blood counts, the spleen's size is normal, the serum EPO level is usually in the normal range or lower, secondary causes of polycythemia are absent, and patients seem healthy. ${ }^{1}$ In the family history, there may be affected relatives requiring regular phlebotomies and/or early deaths in the family due to thrombotic episodes. Sporadic cases of CE have also been reported. ${ }^{5,6}$ Patients are often diagnosed in childhood or early adulthood, following clinical presentations varying from an asymptomatic condition to suffering from headaches, weakness, dizziness, tinnitus, paresthesia, fatigue, blurred vision, and slow mentation. The only physical finding may be plethora. ${ }^{5,6}$

In $\mathrm{CE}$, aside from symptoms that impair quality of life, the development of thromboembolic events is the major complication of the disease. ${ }^{10}$ Such events-stroke, myocardial infarction, deep venous thrombosis, and hypertension-may be life-threatening. ${ }^{5,6}$

The presence of high Hgb and Hct levels should be confirmed in multiple blood counts taken at different times under normal hydration when the patient has no diarrhea and/or inadequate fluid intake or history of using diuretics. If absolute erythrocytosis remains uncertain, then a red cell mass study and P50 test may be performed. In patients with high EPO levels, the sequencing of globin genes for Hgb variants with high oxygen affinity and hemoglobin electrophoresis is useful for differential diagnosis. ${ }^{5}$

Despite all of those findings, data representing children and adolescents with CE in the English-language literature on the topic remain thin, and EPOR mutations are rarely identified in patients. ${ }^{11}$ In the study presented here, our aim in examining those patients was to raise awareness among pediatricians and pediatric hematologists about $\mathrm{CE}$ in order to improve patients' quality of life and prevent potential thrombosis in adolescence and adulthood.

\section{METHODS}

In a single-center, retrospective study approved by the institution's Research Ethics Committee, patients less than 18 years old who were diagnosed with $\mathrm{CE}$ and followed up in a pediatric hematology outpatient unit formed the sample. The paper-based files and electronic medical records of all such patients diagnosed with CE from 2000 to 2020 were evaluated by pediatric hematologists to document demographic characteristics, presenting symptoms, family history, the findings of physical examinations, and the results of laboratory tests. The center's routine practice also entailed screening full blood counts of each patient's parents and siblings. Capillary oxygen saturation, venous blood gas, and serum EPO levels, along with the results of $\mathrm{Hgb}$ electrophoresis and JAK2 mutation analysis, were recorded as well. Phlebotomy practices and any complications of the disease during follow-up were assessed. During the study, patients who had aged beyond 20 years and been transferred to adult departments were telephoned to gather up-to-date medical data. 
Erythrocytosis was defined as having hemoglobin levels exceeding the age- and gender-adjusted 99th percentile or an increase of $2 \mathrm{~g} / \mathrm{dL}$ in hemoglobin levels from baseline. ${ }^{3} \mathrm{Hgb}$ percentile curves were used to evaluate age- and gender-adjusted values, ${ }^{12}$ while for diagnosis, full blood counts were performed at least twice at different times. No patients had serum EPO levels exceeding laboratory reference levels. Patients with thrombocytosis, leukocytosis, and/or splenomegaly were excluded from the sample, as were patients with secondary causes of erythrocytosis (e.g., cardiac, pulmonary, renal, and hepatic disorders and malignancies) according to their history, physical examinations, capillary oxygen saturation analysis, venous blood gas measurement, and serum levels. In patients who reported any bleeding-related symptoms, prothrombin time, activated partial thromboplastin time, and bleeding time assays were also performed.

Statistical analysis was performed using IBM SPSS version 22.0. Categorical variables were analyzed as percentages, and quantitative data were recorded as $M \pm S D$ and median using descriptive statistics.

\section{RESULTS}

From 2000 to 2020, 40 patients were diagnosed with erythrocytosis at the center, in a male-to-female ratio of 7 to 1 . Their mean age was $15.31 \pm 2.49$ years $(8.34-17.92)$ years at diagnosis and $17.92 \pm 2.96$ years (11.43-27.17) at data collection, and the median follow-up time was 9 months, ranging from 6 months to 13.80 years. Thirty-two (80.0\%) patients had been referred in the past 24 months due to full-blood count screening performed in schools. In terms of family relatedness, two of the patients were siblings. Table 1 summarizes the demographic and laboratory characteristics of the patients.

The medical histories of the patients involved no other diseases, and patients generally seemed to be healthy. Seventeen families $(43.58 \%)$ had relatives with erythrocytosis, or relatives who regularly received phlebotomies, and in seven and five families, fathers and siblings were also affected, respectively. In 11 families (28.20\%), 15-48-year-old relatives had experienced myocardial infarction, stroke, and/or sudden death.

Physical examination revealed that $14(35.0 \%)$ patients had plethora, while none had splenomegaly or any other pathological findings. The Hgb levels, age, and sex of the patients at diagnosis appear in Figure 1; mean $\mathrm{Hgb}$ at diagnosis was $17.40 \pm 1.34 \mathrm{~g} / \mathrm{dL}(14.63-23.0 \mathrm{~g} / \mathrm{dL})$. All patients' leukocyte counts, platelet counts, venous blood gas levels, capillary oxygen saturation levels (>95\%), and results of Hgb electrophoresis (i.e., with high-performance liquid chromatography) were within normal limits. To analyze EPOR mutations, the blood samples of the first seven patients had been sent to Portugal, but results revealed only a previously identified heterozygous EPOR nonsense mutation c.1316G $>$ A (p.Trp439Term) in two siblings. ${ }^{13}$ No patient exhibited a JAK2mutation or splenomegaly, thrombocytosis and/or leukocytosis which are characteristic findings of myeloproliferative disease.

Recurrent phlebotomies (i.e., 1-12 times/year) had been performed on demand in all patients in the presence of symptoms of hyperviscosity, and all patients had reported symptom relief after the procedure (Table 1). Many patients had been prescribed acetylsalicylic acid as an antiaggregant but had demonstrated poor compliance. All patients had been informed about potential bleeding and thrombosis at diagnosis, and good hydration, an active lifestyle, and abstinence from smoking, mountain climbing, and scuba diving had been recommended. No thrombotic episodes had occurred in any patients during follow-up.

Symptoms and signs of $\mathrm{CE}$ detected in the patients are listed in Table 2. The most common presenting symptoms were headache $(80.0 \%)$, numbness and tingling in the hands and feet $(45.0 \%)$, and pruritus (37.5\%). At least one gastrointestinal symptom (e.g., nausea, vomiting, abdominal pain, and rectal bleeding) was reported in $40.0 \%$ of patients. Gastrointestinal symptoms were prominent, and patients reported visiting numerous pediatricians for years in search of a diagnosis. Six cases of CE (15.0\%), despite being asymptomatic at diagnosis, were detected during routine whole blood screening.

\section{DISCUSSION}

Full blood counts are a frequently ordered laboratory assay for pediatric patients, in which physicians evaluating Hgb generally focus on the presence of anemia. The normal level of Hgb varies with age and sex in childhood, however, and physicians are not always aware of normal Hgb levels in children. Beyond that, 
symptoms of $\mathrm{CE}$ are non-specific, and in some asymptomatic patients, erythrocytosis is an incidental laboratory finding. ${ }^{5}$ In our study, erythrocytosis was indeed incidentally detected in six asymptomatic patients, and headache was the most common symptom. In the literature, gastrointestinal symptoms such as nausea, vomiting, abdominal pain, and rectal bleeding are not emphasized among symptoms of hyperviscosity. In the present study, some patients with gastrointestinal symptoms had been followed up in a pediatric gastroenterology outpatient clinic where Hgb levels had been overlooked. Patients with minor transient rectal bleeding had been referred to the hematology unit for the evaluation of bleeding disorders. Previously, in a sample of adults with idiopathic erythrocytosis, $4.8 \%$ suffered from bleeding: one from gastrointestinal bleeding and six with minor hemorrhaging. In the same sample, the incidence of bleeding was lower than among patients with $\mathrm{PV}$, and bleeding did not increase with the use of low-dose aspirin. ${ }^{14}$ Minor, self-limited mucosal bleeding (e.g., epistaxis and gingival bleeding) also ranked among the symptoms of three patients at presentation, although coagulation assays and platelet counts revealed normal results.

Most patients $(87.5 \%)$ in our study were males, and research suggests that testosterone stimulates EPO production. ${ }^{15}$ Some female adolescents in our study had reported a drop in the frequency of symptoms of hyperviscosity and requirement of phlebotomy after menarche, likely due to menstrual blood loss. None of the patients' mothers had erythrocytosis, although their histories included thrombotic episodes in both female and male maternal and paternal relatives. Most (70\%) patients had been at least 15 years old at diagnosis, whereas the youngest patient had been only eight years of age. The Hgb levels of adolescents generally range from 16.50 to $18.00 \mathrm{~g} / \mathrm{dL}$, and only three patients had shown values ranging from 20.0 to $23.0 \mathrm{~g} / \mathrm{dL}$.

Among other results, $43.58 \%$ of patients had a family history of erythrocytosis. ${ }^{5,15}$ In one family, a 10 -yearold girl presented with a 2-year history of headache, abdominal ache, nausea, recurrent rectal bleeding, and epistaxis, coupled with an $\mathrm{Hgb}$ level of $16.3 \mathrm{~g} / \mathrm{dL}$. Her mother, a physician, had visited multiple specialists but never received a diagnosis. Her mother had a normal Hgb level $(12.0 \mathrm{~g} / \mathrm{dL})$ but experienced an unprovoked stroke at the age of 40 years. No risk factor of thrombophilia or acquired risk factor (e.g., smoking, obesity, or diabetes mellitus) could be detected. The maternal grandfather also had erythrocytosis that required regular phlebotomies, but genetic analysis revealed no EPOR mutation. It is suggested that the mother experienced the incomplete penetrance of a dominant mutation not already identified. In earlier work, a pathogenic EPOR variant was identified with sequence analysis in only $12 \%-15 \%$ of patients with CE. ${ }^{16-18}$ In our series, family history of erythrocytosis was present in $43.58 \%$ of patients, and more than half of the patients may have had de novo mutations..$^{5,6}$ The frequency of consanguineous marriage in our series was not higher than in Turkey's general population, although recessive mutations are possible.

A normal or low serum EPO level excludes secondary causes of polycythemia associated with hypoxia, but not PV. ${ }^{2}$ Serum EPO levels below $2.9 \mathrm{mU} / \mathrm{mL}$ are specific and moderately sensitive $(92 \%$ and $64 \%$, respectively) to $\mathrm{PV}$, while levels exceeding $15.1 \mathrm{mU} / \mathrm{mL}$, albeit also specific, are largely insensitive (98\% and $47 \%$, respectively) to secondary erythrocytosis. ${ }^{19}$ In our study, serum EPO levels ranged between 2.65 and $16.9 \mathrm{mU} / \mathrm{mL}$, and 31 patients' EPO levels were within the normal range of laboratory reference values. Before 20 years of age, PV is quite rare compared with $\mathrm{CE} .{ }^{20}$ During the 20 -year period of our retrospective study, only one adolescent with PV was diagnosed in pediatric hematology. All 40 patients were referred from a population of roughly 2 million in that period.

The medical histories and physical examinations of the patients did not reveal any other disease. Tissue oxygenation shown by capillary pulse oximetry and venous blood gas analysis excluded hypoxia due to cardiopulmonary disease. All patients had capillary oxygen saturation exceeding 95\%. Although invasive, arterial oxygen saturation is a more sensitive indicator of tissue hypoxia; it is less than $92 \%$ in hypoxia except in cases of chronic carbon monoxide poisoning, smoking, Hgb with high oxygen affinity, and obstructive sleep apnea. ${ }^{1,21,22}$ Arterial oxygen saturation must be performed in patients with elevated EPO levels.

The management of CE in childhood is not evidence-based. Phlebotomy and low-dose acetylsalicylic acid are recommended in symptomatic patients and patients with any past thrombotic episode or with relatives who have experienced such episodes. ${ }^{23}$ Our series did not contain any thrombotic events, and no patient was older than 27 years at data collection. Diagnosis and treatment, along with heeding recommendations about 
lifestyle, can prevent thrombosis. The risk of thrombosis seems to increase with age, although family histories in our series indicated myocardial infarction in 20-year-old relative and sudden deaths in 15- and 18-yearold relatives. Thrombotic events in adults with $\mathrm{CE}$ are widely documented and known to be less common than in patients with secondary erythrocytosis. ${ }^{24}$ Meanwhile, the risk of thrombosis during childhood and adolescence remains unclear. ${ }^{25}$ Phlebotomy is recommended to maintain Hct levels less than $45 \%$ depending on experience in PV. ${ }^{26}$ In our study, all patients had undergone phlebotomy with intervals depending on their needs and the development of symptoms of hyperviscosity. Although some had refused phlebotomy because they claimed to be well and believed that the procedure makes no difference, with age they confessed that they indeed had symptoms but had refused phlebotomy for fear of pain caused by the needle. Family histories included stroke, myocardial infarction, and sudden death but without any diagnosis of CE in most relatives aged 15-48 years. Pulmonary hypertension has also been reported in $\mathrm{CE}$, and screening for the complication is recommended during follow-up. ${ }^{27}$

Limitation of the study: We consider that our patients had EPOR mutations, and erythroid progenitors are hypersensitive to EPO. Although the means to analyze EPOR mutations are unavailable in the country where we conducted the study and could not be performed in most patients, we know that many mutations have remained unidentified.

\section{CONCLUSION}

Pediatric patients with CE may also present with gastrointestinal symptoms (e.g., nausea, vomiting, abdominal pain, and rectal bleeding) in addition to common symptoms of erythrocytosis. Plethora was evident in fewer than half of our sample's patients. To detect CE, physicians should carefully assess Hgb and Htc levels in light of age- and sex-adjusted normal values in children. Although phlebotomies and aspirin increase quality of life and may prevent thrombosis, family histories suggest that thromboembolic complications, even fatal ones, may develop in adolescents.

Conflict of Interest Statement: There is no conflict of interest or financial disclousure.

\section{Acknowledgement:}

Thanks to Oliveira AC and Bento C from University Hospital Coimbra, Portugal for genetic study of EPOR mutations of seven patients.

\section{REFERENCES}

1. Bento C, McMullin MF, Percy M, Cario H. Primary Familial and Congenital Polycythemia. 2016 Nov 10. In: Adam MP, Ardinger HH, Pagon RA, et al., editors. GeneReviews [Internet]. Seattle (WA): University of Washington, Seattle; 1993-2020. Bookshelf URL:https://www.ncbi.nlm.nih.gov/books/

2. McMullin MF, Bareford D, Campbell P, et al. Guidelines for the diagnosis, investigation and management of polycythaemia/erythrocytosis. Br J Haematol 2005;130(2):174-195.

3. Cario H, McMullin MF, Bento C, et al. Erythrocytosis in children and adolescents-classification, characterization, and consensus recommendations for the diagnostic approach. Pediatr Blood Cancer 2013; 60: $1734-1738$.

4. Johansson PL, Soodabeh S-K, Kutti J. An elevated venous hemoglobin concentration cannot be used as a surrogate marker for absolute erythrocytosis: a study of patients with polycythaemia vera and apparent polycythaemia. Br J Haematol 2005; 129:701-705.

5. McMullin MF. Congenital erythrocytosis. Int J Lab Hematol. 2016;38 Suppl 1:59-65.

6. Bento C, Percy MJ, Gardie B, et al.; ECE-Consortium. Genetic basis of congenital erythrocytosis: mutation update and online databases. Hum Mutat. 2014;35:15-26.

7. Tefferi A. Diagnostic approach to the patient with polycythemia. Section editor: Larson RA, Deputy editor: Rosmarin AG. Link:https://www.uptodate.com/contents/diagnostic-approach-to-the-patientwith-polycythemia. Last updated: Oct 02, 2020. Topic 7075 Version 27.0

8. Steinberg MH. Genetic disorders of hemoglobin oxygen affinity. Section editors: Mahoney DH, Raby BA., Deputy editor: Tirnauer JS.https://www.uptodate.com/contents/genetic-disorders-of-hemoglobin- 
oxygen-affinityLast updated: Apr 02, 2019. Topic 7164 Version 20.0

9. Patnaik MM, Tefferi A. The complete evaluation of erythrocytosis: congenital and acquired. Leukemia. 2009 May;23(5):834-844.

10. Gordeuk VR, Sergueeva AI, Miasnikova GY, et al. Congenital disorder of oxygen sensing: association of the homozygous Chuvash polycythaemia VHL mutation with thrombosis and vascular abnormalities but not tumors. Blood 2004;103: 3924-3929.

11. Bento C, Almeida H, Maia TM, et al. Molecular study of congenital erythrocytosis in 70 unrelated patients revealed a potential causal mutation in less than half of the cases (Where is/are the missing gene(s)?). Eur J Haematol. 2013 Oct;91(4):361-368.

12. Dallman PR, Siimes MA. Percentile curves for hemoglobin and red cell volume in infancy and childhood. J Pediatr. 1979 Jan;94(1):26-31.

13. Sarper N, Zengin E, Gelen SA. Clinical characteristics of a Turkish family with congenital erythrocytosis due to an EPOR mutation: Is routine phlebotomy indicated in children and adolescents? Turk Pediatri Ars 2020; 55(3): 312-315.

14. Bertozzi I, Ruggeri M, Nichele I, Biagetti G, Cosi E, Randi ML. Thrombotic and hemorrhagic complications in idiopathic erythrocytosis. Am J Hematol. 2017 Nov;92(11):639-641.

15. Bachman E, Travison TG, Basaria S, et al. Testosterone induces erythrocytosis via increased erythropoietin and suppressed hepcidin: evidence for a new erythropoietin/hemoglobin set point. J Gerontol A Biol Sci Med Sci. 2014;69(6):725-735.

16. Rives S, Pahl HL, Florensa L, et al. Molecular genetic analyses in familial and sporadic congenital primary erythrocytosis. Haematologica. 2007;92:674-677.

17. Al-Sheikh M, Mazurier E, Gardie B, et al. A study of 36 unrelated cases with pure erythrocytosis revealed three new mutations in the erythropoietin receptor gene. Haematologica. 2008;93:1072-1075.

18. Kralovics R, Prchal JT. Genetic heterogeneity of primary familial and congenital polycythemia. Am J Hematol. 2001;68:115-121.

19. Messinezy M, Westwood NB, El-Hemaidi I, et al. Serum erythropoietin values in erythrocytoses and in primary thrombocythaemia. Br J Haematol 2002;117:47-53.

20. Cario H, McMullin MF, Pahl HL. Clinical and hematological presentation of children and adolescents with polycythemia vera. Ann Hematol 2009;88:713-719.

21. Moore-Gillon JC, Treacher DF, Gaminara EJ, Pearson TC, Cameron IR. Intermittent hypoxia in patients with unexplained polycythaemia. Br Med J (Clin Res Ed) 1986; 293(6547):588-590.

22. Mithoowani S, Laureano M, Crowther MA, Hillis CM. Investigation and management of erythrocytosis. CMAJ Aug 2020;192(32):913-918.

23. McMullin MFF, Mead AJ, Ali S, et al.; British Society for Haematology Guideline. A guideline for the management of specific situations in polycythaemia vera and secondary erythrocytosis: A British Society for Haematology Guideline. Br J Haematol. 2019 Jan;184(2):161-175.

24. Gordeuk VR, Key NS, Prchal JT. Re-evaluation of hematocrit as a determinant of thrombotic risk in erythrocytosis. Haematologica. 2019 Apr;104(4):653-658.

25. Tefferi A, Barbui T. Polycythemia vera and essential thrombocythemia: 2015 update on diagnosis, riskstratification and management [published correction appears in Am J Hematol. 2015 Sep;90(9):849]. Am J Hematol 2015; 90: 162-73.

26. Marchioli, R., Finazzi, G., Specchia, et al. (2013) Cardiovascular events and intensity of treatment in polycythemia vera. New England Journal of Medicine, 2013; 368: 22-33.

27. Gale DP, Harten SK, Reid CD, Tuddenham GD, Maxwell PH. Autosomal dominant erythrocytosis and pulmonary arterial hypertension associated with HIF2? mutation. Blood 2008; 112:919-2122.

\section{Figure Legend}

Figure 1. Distribution of hemoglobin values of patients at diagnosis.

\section{Hosted file}

Table 1- Demographic and laboratory characteristics of patients.pdf available at https: 
//authorea.com/users/407411/articles/517730-clinical-characteristics-of-pediatricpatients-with-congenital-erythrocytosis-a-single-center-study

\section{Hosted file}

Table 2.Symptoms and signs of the 40 children and adolescents with congenital erythrocytosis.pdf available at https://authorea.com/users/407411/articles/517730-clinical-characteristics-ofpediatric-patients-with-congenital-erythrocytosis-a-single-center-study

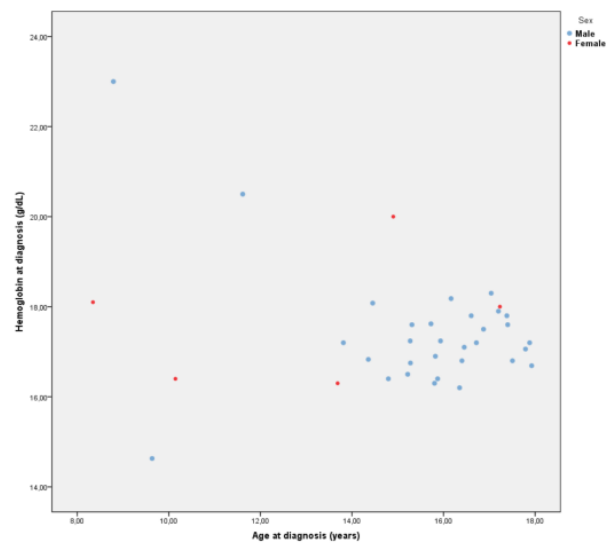

